\title{
Relaxed Quasimonotone Operators and Relaxed Quasiconvex Functions ${ }^{1}$
}

M. R. BAI ${ }^{2}$ AND N. HADJISAVVAS ${ }^{3}$

\footnotetext{
${ }^{1}$ This work is supported by NNSF of China (10571046) and by the GSRT of Greece (06FR-062).

${ }^{2}$ Associate Professor, College of Mathematics and Econometrics, Hunan University, Changsha, Hunan 410082, P.R. China.

${ }^{3}$ Professor, Department of Product and Systems Design Engineering, University of the Aegean, Hermoupolis, 84100, Syros, Greece.
} 


\begin{abstract}
In this paper, we introduce the class of multivalued relaxed $\mu$ quasimonotone operators and establish the existence of solutions of variational inequalities for such operators. This result is compared with a recent result of Bai et al on densely relaxed pseudomonotone operators. A similar comparison regarding an existence result of Luc on densely pseudomonotone operators is provided. Also, we introduce a broad class of functions, called relaxed quasiconvex functions, and show that they are characterized by the relaxed $\mu$ quasimonotonicity of their subdifferentials. The results strengthen a variety of other results in the literature.
\end{abstract}

Key Words. Variational inequality; Relaxed $\mu$ quasimonotone operator; Relaxed quasiconvex function; Subdifferential; Dag subdifferential. 


\section{Introduction}

Let $X$ be a normed space with norm $\|\cdot\|$ and dual space $X^{*}$. Let $K$ be a nonempty convex subset of $X$ and $T: K \rightarrow 2^{X^{*}} \backslash\{\emptyset\}$ a multivalued operator. The Stampacchia variational inequality problem for $T$ is to find $x \in K$ such that

$$
\forall y \in K, \exists x^{*} \in T(x):\left\langle x^{*}, y-x\right\rangle \geq 0 .
$$

The usual assumptions for proving the existence of solutions for (1) involve some kind of upper semicontinuity, and some kind of generalized monotonicity for the operator $T$. Various generalized monotonicity assumptions were used, such as: $T$ is pseudomonotone (Refs. 1, 2 ); $T$ is quasimonotone, single valued, and $K$ has inner points (Ref. 3 ); $T$ is properly quasimonotone (Ref. 4 ); $T$ is single-valued and densely pseudomonotone (Ref. 5 ); $T$ is quasimonotone (without any assumption on the existence of inner points) (Ref. 6). A different kind of generalization, namely relaxed monotonicity (or else weak monotonicity, or global hypomonotonicity) was considered by various authors in relation with algorithms for finding a solution of variational inequalities or equilibrium problems; see, e.g., Refs. 7, 8 . However, in these papers existence of a solution of the variational inequality was assumed rather than shown.

Recently, those different generalizations were combined and existence of solutions of (1) was shown for the case of single valued, densely relaxed $\mu$ pseudomonotone operators (Refs. 9, 10 ).

In the present paper we will introduce the broader class of multivalued relaxed $\mu$ quasimonotone operators and establish the existence of solutions of (1) for such operators. This is done in Section 2, where we also compare our main result with a recently obtained result for densely relaxed pseudomonotone operators (Ref. 10 ). With an analogous method, we investigate the relation of densely pseudomonotone operators introduced by Luc (Ref. 5 ) to properly quasimonotone operators. In Section 3 we introduce a broad class of functions, called relaxed $\mu$ quasiconvex functions, and show that they are characterized by the relaxed $\mu$ quasimonotonicity of their subdifferentials.

We fix the notation and introduce some definitions. Given $x, y \in X$, we will denote by $[x, y]$ the line segment

$$
\{x+t(y-x): t \in[0,1]\} \text {. }
$$

A multivalued operator $T: K \rightarrow 2^{X^{*}} \backslash\{\emptyset\}$ is called upper hemicontinuous if its restriction to line segments of $K$ is upper semicontinuous with respect to the weak ${ }^{*}$ topology of $X^{*}$. If $T$ is single valued, it is called hemicontinuous 
if its restriction to line segments of $K$ is continuous with respect to the weak ${ }^{*}$ topology of $X^{*}$. We denote by $S(T, K)$ the set of solutions of the variational inequality (1):

$$
x \in S(T, K) \Longleftrightarrow x \in K \text { and } \forall y \in K, \exists x^{*} \in T(x):\left\langle x^{*}, y-x\right\rangle \geq 0 .
$$

Also, we denote by $\operatorname{LM}(T, K)$ the set of local solutions (Ref. 6 ) of the Minty variational inequality

$x \in L M(T, K) \Longleftrightarrow x \in K$ and $\exists U: \forall y \in K \cap U, \forall y^{*} \in T(y),\left\langle y^{*}, y-x\right\rangle \geq 0$

where $U$ is a neighborhood of $x$.

The following definition generalizes Definition 2.1.(ii) of Ref. 10 to the multivalued case. Let $\mu>0$ be given.

Definition 1.1 A multivalued operator $T: K \rightarrow 2^{X^{*}} \backslash\{\emptyset\}$ is called relaxed $\mu$ quasimonotone if for all $x, y \in K$ and $x^{*} \in T(x), y^{*} \in T(y)$, the following implication holds:

$$
\left\langle x^{*}, y-x\right\rangle>0 \Rightarrow\left\langle y^{*}, y-x\right\rangle \geq-\mu\|y-x\|^{2} .
$$

We also introduce the relaxed Minty variational inequality

$$
\forall y \in K, \forall y^{*} \in T(y),\left\langle y^{*}, y-x\right\rangle \geq-\mu\|y-x\|^{2}
$$

and denote by $R M(T, K)$ the set of $x \in K$ that satisfy (2).

We recall that a set-valued mapping $G: K \rightarrow 2^{X}$ is said to be a KKM mapping if for any $\left\{x_{1}, \ldots, x_{n}\right\} \subset K, \operatorname{co}\left\{x_{1}, \ldots, x_{n}\right\} \subset \bigcup_{i=1}^{n} G\left(x_{i}\right)$, where $\operatorname{co}\left\{x_{1}, \ldots, x_{n}\right\}$ denotes the convex hull of $x_{1}, \ldots, x_{n}$. For the convenience of the reader we reproduce the fundamental lemma of Ky Fan on KKM mappings (Ref. 11).

Lemma 1.1 Assume that $K$ is a nonempty compact convex subset of a Hausdorff topological vector space $X$. Let $K_{0} \subseteq K$ be nonempty and $G$ : $K_{0} \rightarrow 2^{K}$ a KKM mapping. If $G(x)$ is closed in $X$ for each $x$ in $K_{0}$, then

$$
\bigcap_{x \in K_{0}} G(x) \neq \emptyset
$$

\section{Existence Result}

We introduce the following definition. Let $\mu>0$ be given. 
Definition 2.1 An operator $T: K \rightarrow 2^{X^{*}} \backslash\{\emptyset\}$ is said to be properly relaxed $\mu$ quasimonotone if for all $x_{1}, \cdots, x_{n} \in K$, and all $x \in \operatorname{co}\left\{x_{1}, \cdots, x_{n}\right\}$, there exists $i \in\{1, \cdots, n\}$ such that

$$
\forall x^{*} \in T\left(x_{i}\right),\left\langle x^{*}, x_{i}-x\right\rangle \geq-\mu\left\|x_{i}-x\right\|^{2} .
$$

Proposition 2.1 If $T$ is properly relaxed $\mu$ quasimonotone then it is relaxed $\mu$ quasimonotone.

Proof. Let $x, y \in K$ and $x_{0}^{*} \in T(x)$ be such that $\left\langle x_{0}^{*}, y-x\right\rangle>0$. Set $x_{t}=x+t(y-x), t \in(0,1)$. Since $T$ is $\mu$ properly relaxed quasimonotone we have either

$$
\left\langle x^{*}, x-x_{t}\right\rangle \geq-\mu\left\|x-x_{t}\right\|^{2}, \forall x^{*} \in T(x), \forall t \in(0,1)
$$

or

$$
\left\langle y^{*}, y-x_{t}\right\rangle \geq-\mu\left\|y-x_{t}\right\|^{2}, \forall y^{*} \in T(y), \forall t \in(0,1) .
$$

However, (3) implies that

$$
\left\langle x_{0}^{*}, y-x\right\rangle \leq \mu t\|y-x\|^{2} \forall t \in(0,1)
$$

which clearly contradicts $\left\langle x_{0}^{*}, y-x\right\rangle>0$. Thus (4) holds. Taking the limit in (4) as $t \rightarrow 0$ we deduce that

$$
\left\langle y^{*}, y-x\right\rangle \geq-\mu\|y-x\|^{2}, \forall y^{*} \in T(y)
$$

i.e., $T$ is relaxed $\mu$ quasimonotone.

Lemma 2.1 Let $K$ be a nonempty convex subset of a normed space $X$. If $T: K \rightarrow 2^{X^{*}} \backslash\{\emptyset\}$ is upper hemicontinuous with weakly* compact values, then $R M(T, K) \subseteq S(T, K)$.

Proof. Assume to the contrary that there exists $x \in R M(T, K)$ such that $x \notin S(T, K)$. Then there exists $y \in K$ such that $\left\langle x^{*}, y-x\right\rangle<0$ for all $x^{*} \in T(x)$. Since $T(x)$ is weakly* compact, there exists $\varepsilon>0$ such that $\left\langle x^{*}, y-x\right\rangle<-\varepsilon$ for all $x^{*} \in T(x)$.

If we set

$$
V=\left\{x^{*} \in X^{*}:\left\langle x^{*}, y-x\right\rangle<-\varepsilon\right\}
$$

then $V$ is an weak* ${ }^{*}$ open set such that $T(x) \subseteq V$. Set $x_{t}=t y+(1-t) x$, $t \in(0,1]$. By upper hemicontinuity, there exists $\delta>0$ such that $T\left(x_{t}\right) \subseteq V$ for all $t \in(0, \delta)$, i.e.,

$$
\left\langle x^{*}, y-x\right\rangle<-\varepsilon, \quad, \forall t \in(0, \delta), \forall x^{*} \in T\left(x_{t}\right) .
$$


On the other hand, since $x \in R M(T, K)$, there exists a constant $\mu$ such that for all $t \in(0, \delta)$ and all $x^{*} \in T\left(x_{t}\right)$ we have

$$
\begin{aligned}
\left\langle x^{*}, x_{t}-x\right\rangle & =t\left\langle x^{*}, y-x\right\rangle \\
& \geq-\mu\left\|x_{t}-x\right\|^{2} \\
& =-\mu t^{2}\|y-x\|^{2}
\end{aligned}
$$

i.e.,

$$
\left\langle x^{*}, y-x\right\rangle \geq-\mu t\|y-x\|^{2} .
$$

It is clear that for $t$ small enough, (5) contradicts (6). Therefore $R M(T, K) \subseteq$ $S(T, K)$.

We deduce that the following alternative holds for every relaxed $\mu$ quasimonotone operator.

Proposition 2.2 Let $K$ be a nonempty, convex subset of a normed space $X$ and let $T: K \rightarrow 2^{X^{*}} \backslash\{\emptyset\}$ be relaxed $\mu$ quasimonotone. Then, one of the following assertions holds:

(i) $T$ is properly relaxed $\mu$ quasimonotone;

(ii) $\operatorname{LM}(T, K) \neq \emptyset$.

Proof. Suppose that $T$ is not properly relaxed $\mu$ quasimonotone. Then there exist $x_{i} \in K, x_{i}^{*} \in T\left(x_{i}\right), i=1, \ldots, n$ and $x \in \operatorname{co}\left\{x_{1}, x_{2}, \ldots, x_{n}\right\}$ such that

$$
\left\langle x_{i}^{*}, x_{i}-x\right\rangle<-\mu\left\|x-x_{i}\right\|^{2}, \quad i=1, \ldots, n .
$$

Since the functions $\left\langle x_{i}^{*}, x_{i}-\cdot\right\rangle+\mu\left\|\cdot-x_{i}\right\|^{2}$ are continuous, there exists a neighborhood $U$ of $x$ such that for every $z \in K \cap U$,

$$
\left\langle x_{i}^{*}, x_{i}-z\right\rangle<-\mu\left\|z-x_{i}\right\|^{2} .
$$

Since $T$ is relaxed $\mu$ quasimonotone, it follows that for every $i=1, \ldots, n$ and every $z^{*} \in T(z)$,

$$
\left\langle z^{*}, z-x_{i}\right\rangle \geq 0
$$

Using $x \in \operatorname{co}\left\{x_{1}, x_{2}, \ldots, x_{n}\right\}$ we deduce that

$$
\forall z \in K \cap U, \forall z^{*} \in T(z), \quad\left\langle z^{*}, z-x\right\rangle \geq 0 .
$$

This means that $x \in L M(T, K)$ thus $\operatorname{LM}(T, K) \neq \emptyset$.

The above alternative entails our main existence result.

Proposition 2.3 Let $K$ be a nonempty, compact, convex subset of a normed space $X$ and let $T: K \rightarrow 2^{X^{*}} \backslash\{\emptyset\}$ be relaxed $\mu$ quasimonotone and upper hemicontinuous with weakly* compact values. Then $S(T, K) \neq \emptyset$. 
Proof. According to the preceding proposition, we have either $\operatorname{LM}(T, K) \neq$ $\emptyset$ or that $T$ is properly relaxed $\mu$ quasimonotone. If $L M(T, K) \neq \emptyset$ then we know that $S(T, K) \neq \emptyset$ (Ref. 6 ). On the other hand, if $T$ is properly relaxed $\mu$ quasimonotone, define the multivalued mapping $G: K \rightarrow 2^{X^{*}} \backslash\{\emptyset\}$ by

$$
G(x)=\left\{y \in K:\left\langle x^{*}, x-y\right\rangle \geq-\mu\|x-y\|^{2}, \forall x^{*} \in T(x)\right\} .
$$

For every $x_{1}, \ldots, x_{n} \in K$ and $y \in \operatorname{co}\left\{x_{1}, x_{2}, \ldots, x_{n}\right\}$, proper relaxed quasimonotonicity implies that $y \in \bigcup_{i=1}^{n} G\left(x_{i}\right)$. In addition, for each $x \in$ $K, G(x)$ is closed; thus, if $K$ is compact, then for each $x \in K, G(x)$ is also compact. By Lemma 1.1, one has $\bigcap_{x \in K} G(x) \neq \emptyset$, which implies that $R M(T, K) \neq \emptyset$. Finally by Lemma 2.1 we obtain again $S(T, K) \neq \emptyset$.

Below we derive a useful consequence for problem (1) over unbounded sets. We will make use of the following coercivity condition for an operator $T: K \rightarrow 2^{X^{*}} \backslash\{\emptyset\}:$

$$
\begin{aligned}
& \exists \rho>0 \text { such that } \forall x \in K \backslash \bar{B}(0, \rho), \\
& \exists y \in K \text { such that }\|y\|<\|x\| \text { and }\left\langle x^{*}, x-y\right\rangle \geq 0, \forall x^{*} \in T(x)
\end{aligned}
$$

Theorem 2.1 Let $K$ be a convex unbounded subset of a normed space $X$ and let $T: K \rightarrow 2^{X^{*}} \backslash\{\emptyset\}$ be upper hemicontinuous and relaxed $\mu$ quasimonotone on $K$ with weakly* compact values, satisfying coercivity condition (C). Suppose that there exists $\rho^{\prime}>\rho$ such that $K \cap \bar{B}\left(0, \rho^{\prime}\right)$ is nonempty and compact. Then $S(T, K) \neq \emptyset$.

Proof. Set $K_{\rho^{\prime}}=K \cap \bar{B}\left(0, \rho^{\prime}\right)$. By Proposition 2.3, the set $S\left(T, K_{\rho^{\prime}}\right)$ is nonempty. Choose $x_{0} \in S\left(T, K_{\rho^{\prime}}\right)$. According to coercivity condition (C), there exists $y_{0} \in B\left(0, \rho^{\prime}\right) \cap K$ such that

$$
\left\langle x^{*}, x_{0}-y_{0}\right\rangle \geq 0, \forall x^{*} \in T\left(x_{0}\right) .
$$

(If $\left\|x_{0}\right\|<\rho^{\prime}$ we can take $y_{0}=x_{0}$ ). Now, for every $y \in K$, choose $t \in(0,1)$ such that $(1-t) y+t y_{0} \in K_{\rho^{\prime}}$. Since $x_{0} \in S\left(T, K_{\rho^{\prime}}\right)$, there exists $x^{*} \in T\left(x_{0}\right)$ such that

$$
\left\langle x^{*},(1-t) y+t y_{0}-x_{0}\right\rangle \geq 0 .
$$

Multiplying (8) by $t$ and adding to (9) yields

$$
\left\langle x^{*}, y-x_{0}\right\rangle \geq 0
$$

which means that $x_{0} \in S(T, K)$.

Note that, in Theorem 2.1, the condition of compactness of $K \cap \bar{B}\left(0, \rho^{\prime}\right)$ is satisfied automatically if $K$ is locally compact. 
In order to compare our results with those existing in the literature, we recall some definitions. Given a convex subset $K$ of $X$, a set $K_{0} \subseteq K$ is called segment-dense in $K$ if for every $x \in K$ there exists $x_{0} \in K_{0}$ such that $x$ is a cluster point of the set $\left[x, x_{0}\right] \cap K_{0}$. Given $\mu>0$, a single-valued operator $T: K \rightarrow X^{*}$ is called relaxed $\mu$ pseudomonotone at $x \in K$ if for every $y \in K$ the following implication holds

$$
\langle T(x), y-x\rangle \geq 0 \Rightarrow\langle T(y), y-x\rangle \geq-\mu\|x-y\|^{2} .
$$

The operator $T$ is called densely relaxed $\mu$ pseudomonotone if there exists a segment-dense set $K_{0} \subseteq K$ such that $T$ is relaxed $\mu$ pseudomonotone at every $x \in K_{0}$. In Ref. 10, it was shown that if $K$ is compact and $T$ is single valued, hemicontinuous and densely relaxed $\mu$ pseudomonotone, then the variational inequality problem has a solution. In order to compare with Proposition 2.3 we first show:

Proposition 2.4 Assume that the operator $T: K \rightarrow X^{*}$ is densely relaxed $\mu$ pseudomonotone and hemicontinuous. Then $T$ is properly relaxed $\mu$ quasimonotone.

Proof. Suppose that $T$ is not properly relaxed $\mu$ quasimonotone. Then there exist $x_{1}, x_{2}, \ldots x_{n} \in K, x=\sum_{i=1}^{n} \lambda_{i} x_{i}$ with $\lambda_{i}>0$ and $\sum_{i=1}^{n} \lambda_{i}=1$ such that

$$
-\mu\left\|x-x_{i}\right\|^{2}>\left\langle T\left(x_{i}\right), x_{i}-x\right\rangle, i=1,2, \ldots n .
$$

By assumption, there exists $v \in X$ and a sequence $\left(t^{k}\right)_{k \in \mathbb{N}}$ converging to zero, such that $T$ is relaxed $\mu$ pseudomonotone at $x^{k}=x+t^{k} v, k \in \mathbb{N}$. From (11) follows that for $k$ sufficiently large,

$$
-\mu\left\|x^{k}-x_{i}\right\|^{2}>\left\langle T\left(x_{i}\right), x_{i}-x^{k}\right\rangle, i=1,2, \ldots n .
$$

Since $T$ is relaxed $\mu$ pseudomonotone at $x^{k}$ we deduce that

$$
\left\langle T\left(x^{k}\right), x_{i}-x^{k}\right\rangle<0
$$

For $k$ sufficiently large one has $x^{k} \in \operatorname{co}\left\{x_{1}, x_{2}, \ldots x_{n}\right\}$. Then (12) implies $\left\langle T\left(x^{k}\right), x^{k}-x^{k}\right\rangle<0$, a contradiction.

As the previous proposition shows, hemicontinuity together with densely relaxed $\mu$ pseudomonotonicity imply that $T$ is properly relaxed $\mu$ quasimonotone. In particular, $T$ is relaxed $\mu$ quasimonotone, thus the existence result in Ref. 10 can be derived from Proposition 2.3. It should be noted that properly relaxed $\mu$ pseudomonotonicity implies that $R M(T, K) \neq \emptyset$ (see the proof of Proposition 2.3), hence the assumptions in Ref. 10 imply that $R M(T, K) \neq \emptyset$. This is not the case when $T$ is only relaxed $\mu$ quasimonotone. 
Likewise, in Ref. 5 a single-valued operator $T: K \rightarrow X^{*}$ is called pseudomonotone at $x \in K$ if for every $y \in K$ the following implication holds:

$$
\langle T(y), x-y\rangle \geq 0 \Rightarrow\langle T(x), x-y\rangle \geq 0 .
$$

The operator is called densely pseudomonotone if it is pseudomonotone for every $x$ in a segment-dense subset $K_{0}$ of $K$. Note that the difference with the definition of dense relaxed $\mu$ pseudomonotonicity is not simply that one takes $\mu=0$. However, by a suitable modification of the proof of Proposition 2.4 we obtain a similar result.

Proposition 2.5 Assume that the operator $T: K \rightarrow X^{*}$ is densely pseudomonotone and hemicontinuous. Then $T$ is properly quasimonotone.

Proof. Suppose that $T$ is not properly $\mu$ quasimonotone. Then there exist $x_{1}, x_{2}, \ldots x_{n} \in K, x=\sum_{i=1}^{n} \lambda_{i} x_{i}$ with $\lambda_{i}>0$ and $\sum_{i=1}^{n} \lambda_{i}=1$ such that

$$
\left\langle T\left(x_{i}\right), x-x_{i}\right\rangle>0, i=1,2, \ldots n .
$$

By assumption, there exist $v_{i} \in X$ and sequences $\left(t_{i}^{k}\right)_{k \in \mathbb{N}}$ converging to 0 as $k \rightarrow+\infty$, such that $T$ is pseudomonotone at $x_{i}^{k}=x_{i}+t_{i}^{k} v$. Set $x^{k}=\sum_{i=1}^{n} \lambda_{i} x_{i}^{k}$. Then $x_{i}^{k} \rightarrow x_{i}$ and $x^{k} \rightarrow x$ strongly and $T\left(x_{i}^{k}\right) \rightarrow T\left(x_{i}\right)$ in the weak* topology as $k \rightarrow+\infty$, thus for $k$ sufficiently large,

$$
\left\langle T\left(x_{i}^{k}\right), x^{k}-x_{i}^{k}\right\rangle>0, i=1,2, \ldots n .
$$

Since $T$ is pseudomonotone at $x_{i}^{k}$ we deduce that

$$
\left\langle T\left(x^{k}\right), x^{k}-x_{i}^{k}\right\rangle>0 .
$$

From the definition of $x^{k}$ we infer that $\left\langle T\left(x^{k}\right), x^{k}-x^{k}\right\rangle>0$, a contradiction.

\section{Relaxed quasiconvex functions}

In this section, nonsmooth relaxed quasiconvex functions will be introduced. We will show that they are characterized by the relaxed quasimonotonicity of their subdifferential.

Throughout this section, $X$ denotes a Banach space with a $\partial$-smooth renorming (Ref. 12). Given a l.s.c. function $f: X \rightarrow \mathbb{R} \cup\{+\infty\}, \partial f$ denotes any subdifferential of $f$ (see Ref. 12 ). We need the following approximate mean value inequality result from Ref. 12 . 
Lemma 3.1 Let $a, b \in X$ with $a \in \operatorname{dom} f$ and $a \neq b$, and let $r \in \mathbb{R}$ be such that $r \leq f(b)$. Then there exist $c \in[a, b], c \neq b$, and sequences $x_{n} \rightarrow c$ and $x_{n}^{*} \in \partial f\left(x_{n}\right)$ such that

$$
\liminf _{n \rightarrow+\infty}\left\langle x_{n}^{*}, c-x_{n}\right\rangle \geq 0 \text { and } \liminf _{n \rightarrow+\infty}\left\langle x_{n}^{*}, b-a\right\rangle \geq r-f(a) .
$$

Definition 3.1 A l.s.c. function $f: X \rightarrow \mathbb{R} \cup\{+\infty\}$ will be called relaxed $\mu$ quasiconvex if for all $x, y \in X$ the following implication holds

$\exists x^{*} \in \partial f(x):\left\langle x^{*}, y-x\right\rangle>0 \Rightarrow \forall z \in[x, y], \quad f(y) \geq f(z)-\mu\|y-z\|\|y-x\|$.

In order to understand the above definition, it is useful to compare it with the characterization of quasiconvex functions in Ref. 13: A l.s.c. function is quasiconvex if and only if

$$
\exists x^{*} \in \partial f(x):\left\langle x^{*}, y-x\right\rangle>0 \Rightarrow \forall z \in[x, y], \quad f(y) \geq f(z) .
$$

Proposition 3.1 Let $X$ be a Banach space with a $\partial$-smooth renorming and $f: X \rightarrow \mathbb{R} \cup\{+\infty\}$ be a l.s.c. function. If $\partial f$ is relaxed $\mu$ quasimonotone, then $f$ is relaxed $\mu$ quasiconvex.

Proof. Suppose that $\partial f$ is relaxed $\mu$ quasimonotone. Let $x \in \operatorname{dom} \partial f$, $y \in \operatorname{dom} f, x \neq y$, and $z \in[x, y], z \neq y$ be such that

$$
f(y)<f(z)-\mu\|y-z\|\|y-x\| \text {. }
$$

Then we can find $\mu_{0}>\mu$ such that

$$
f(z)>f(y)+\mu_{0}\|y-z\|\|y-x\| .
$$

Letting $r=f(y)+\mu_{0}\|y-z\|\|y-x\|$ and applying Lemma 3.1 to $y$ and $z$, we can find $c \in[y, z], c \neq z$ and sequences $x_{n}$ converging to $c$ and $x_{n}^{*} \in \partial f\left(x_{n}\right)$ satisfying

$$
\liminf _{n \rightarrow+\infty}\left\langle x_{n}^{*}, c-x_{n}\right\rangle \geq 0 \quad \text { and } \quad \liminf _{n \rightarrow+\infty}\left\langle x_{n}^{*}, z-y\right\rangle \geq \mu_{0}\|y-z\|\|y-x\|
$$

Letting $u=c+t(z-y)$ with $t>0$, we find

$$
\begin{aligned}
\liminf _{n \rightarrow+\infty}\left\langle x_{n}^{*}, u-x_{n}\right\rangle & \geq \liminf _{n \rightarrow+\infty}\left\langle x_{n}^{*}, c-x_{n}\right\rangle+\liminf _{n \rightarrow+\infty} t\left\langle x_{n}^{*}, z-y\right\rangle \\
& \geq 0+t \mu_{0}\|y-z\|\|y-x\| \\
& =\mu_{0}\|u-c\|\|y-x\| .
\end{aligned}
$$


In particular, taking $u=x$ we deduce

$$
\begin{aligned}
\liminf _{n \rightarrow+\infty}\left\langle x_{n}^{*}, x-x_{n}\right\rangle & \geq \mu_{0}\|x-c\|\|y-x\| \\
& >\mu \lim _{n \rightarrow+\infty}\left\|x-x_{n}\right\|^{2}
\end{aligned}
$$

since $\lim _{n \rightarrow+\infty}\left\|x-x_{n}\right\|=\|x-c\| \leq\|y-x\|$. Hence, for $n$ sufficiently large,

$$
\left\langle x_{n}^{*}, x-x_{n}\right\rangle>\mu\left\|x-x_{n}\right\|^{2} .
$$

By relaxed $\mu$ quasimonotonicity of $\partial f$, we have

$$
\forall x^{*} \in \partial f(x):\left\langle x^{*}, x-x_{n}\right\rangle \geq 0
$$

for $n$ sufficiently large. Letting $n \rightarrow+\infty$, it follows that for all $x^{*} \in \partial f(x)$

$$
\left\langle x^{*}, x-c\right\rangle \geq 0,
$$

and so

$$
\left\langle x^{*}, x-y\right\rangle=[\|x-y\| /\|x-c\|]\left\langle x^{*}, x-c\right\rangle \geq 0 .
$$

Thus, $\partial f$ is relaxed $\mu$ quasiconvex, and the proof is completed.

The converse of Proposition 3.1 also holds for a fairly large class of subdifferentials. Consider the so-called "dag subdifferential" (Ref. 14):

$\partial^{\dagger} f(x):=\left\{x^{*} \in X^{*}:\left\langle x^{*}, v\right\rangle \leq \limsup _{t \searrow 0, w \rightarrow x} \frac{f(w+t(v+x-w))-f(w)}{t}, \forall v \in X\right\}$.

The dag subdifferential is larger than most known subdifferentials. For instance, it is larger than the Gâteaux subdifferential, the upper Dini subdifferential, the Clarke-Rockafellar subdifferential etc. Consequently, it is also larger than the proximal subdifferential and the Fréchet subdifferential.

Proposition 3.2 Assume that $\partial$ is any subdifferential such that $\partial f(x) \subseteq$ $\partial^{\dagger} f(x)$, for every $x \in X$. If $f$ is relaxed $\mu$ quasiconvex, then $\partial f$ is relaxed $\mu$ quasimonotone.

Proof. Obviously, it is sufficient to show that $\partial^{\dagger} f$ is relaxed $\mu$ quasimonotone. Assume that $f$ is relaxed $\mu$ quasiconvex. Let $\left\langle x^{*}, y-x\right\rangle>0$ for some $x^{*} \in \partial^{\dagger} f(x)$. Then $\left\langle x^{*}, w-x\right\rangle>0$ for every $w \in X$ sufficiently close to $y$. Relaxed $\mu$ quasiconvexity of $f$ implies

$$
f(w+t(x-w))-f(w) \leq \mu t\|w-x\|^{2}, \forall t \in[0,1] .
$$


So, for all $y^{*} \in \partial^{\dagger} f(y)$,

$$
\begin{aligned}
\left\langle y^{*}, x-y\right\rangle & \leq \limsup _{t \searrow 0, w \rightarrow y} \frac{f(w+t(x-y+y-w)-f(w)}{t} \\
& \leq \limsup _{t \searrow 0, w \rightarrow y} \mu\|w-x\|^{2} \\
& =\mu\|y-x\|^{2},
\end{aligned}
$$

i.e.,

$$
\left\langle y^{*}, y-x\right\rangle \geq-\mu\|y-x\|^{2},
$$

which implies $\partial^{\dagger} f$ is relaxed $\mu$ quasimonotone. This completes the proof.

Especially, if $f$ is a Gâteaux differentiable function, and if we set $z=x$ in the right-hand side of (13), then from Proposition 3.1 we also get the following property: if $\nabla f$ is relaxed $\mu$ quasimonotone, then there exists a $\nu>0$ such that the following implication holds:

$$
\langle\nabla f(x), y-x\rangle>0 \Rightarrow f(y) \geq f(x)-\nu\|x-y\|^{2} .
$$

(Actually, $\nu=\mu$ ). The following example shows that a differentiable function $f$ may satisfy (14) while $\nabla f$ is not relaxed $\mu$ quasimonotone for any $\mu$.

Example 3.1 Let $f_{1}:[0,+\infty) \rightarrow \mathbb{R}$ be a function with the following properties: $0 \leq f_{1} \leq 1, f_{1}$ is differentiable on $[0,+\infty)$ with $f_{1}^{\prime} \geq 0$ and $f_{1}^{\prime}\left(\frac{1}{n}\right)=n^{3}$. See the end of the example for the construction of such a function. Define $f: \mathbb{R} \rightarrow \mathbb{R}$ by

$$
f(x)=\left\{\begin{array}{c}
-x^{2}\left(1+f_{1}(x)\right), \quad x>0 \\
-x^{2}, \quad x \leq 0 .
\end{array}\right.
$$

This function is differentiable and

$$
f^{\prime}(x)=\left\{\begin{array}{l}
-2 x\left(1+f_{1}(x)\right)-x^{2} f_{1}^{\prime}(x)<0, \quad x>0 \\
0, \quad x=0 \\
-2 x>0, \quad x<0 .
\end{array}\right.
$$

We show that $f$ satisfies (14). Assume that $f^{\prime}(x)(y-x)>0$. If $f^{\prime}(x)>0$, then $x<0$ and $y>x$. If $x<y \leq 0$ then

$$
f(y)=-y^{2}>-x^{2}-2(y-x)^{2} .
$$

If $y>0$ then

$$
f(y)=-y^{2}\left(1+f_{1}(y)\right) \geq-2 y^{2}>-x^{2}-2(y-x)^{2} .
$$


In the second case $f^{\prime}(x)<0$, we have $x>0$ and $y<x$. If $y \leq 0$ then

$$
f(y)=-y^{2}>-x^{2}-(y-x)^{2} \geq-x^{2}\left(1+f_{1}(x)\right)-2(y-x)^{2} .
$$

Finally, if $0<y<x$, then $f_{1}^{\prime} \geq 0$ implies that $f_{1}(y) \leq f_{1}(x)$ thus

$$
f(y)-f(x) \geq\left(x^{2}-y^{2}\right)\left(1+f_{1}(x)\right)>-2(y-x)^{2} .
$$

Thus, (14) holds with $\nu=2$. On the other hand, $f^{\prime}$ is not relaxed $\mu$ quasimonotone for any $\mu$. Indeed, assume that $f^{\prime}$ is relaxed $\mu$ quasimonotone. For every $y>0$ and every $x<0$ one has $f^{\prime}(x)(y-x)>0$ thus we should have

$$
f^{\prime}(y) \geq-\mu(y-x) .
$$

Taking $y=\frac{1}{n}$ we find

$$
f^{\prime}\left(\frac{1}{n}\right) \geq-\frac{1}{n^{2}} f_{1}^{\prime}\left(\frac{1}{n}\right)=-n \rightarrow-\infty .
$$

Thus it is clear that (15) is not possible.

A note on the construction of the function $f_{1}$ : set $I_{n}=\left[\frac{1}{n}-\frac{1}{4 n^{5}}, \frac{1}{n}+\frac{1}{4 n^{5}}\right]$. These intervals are disjoint. Let $g$ be any continuous nonnegative function such that $g=0$ on $\mathbb{R} \backslash \bigcup_{n \geq 1} I_{n}$ and on each $I_{n}$ takes its maximum value at $\frac{1}{n}$ with $g\left(\frac{1}{n}\right)=n^{3}$. This $g$ is integrable with $\int_{\mathbb{R}} g \leq \sum_{n \geq 1} n^{3} \frac{1}{2 n^{5}}=\frac{\pi^{2}}{12}<1$. Finally, define $f_{1}:[0,+\infty) \rightarrow \mathbb{R}$ by

$$
f_{1}(x)=\int_{0}^{x} g(t) d t .
$$

This function $f_{1}$ has all desired properties. 


\section{References}

1. Cottle, R. W., and Yao, J. C., Pseudomonotone Complementarity Problems in Hilbert Spaces, Journal of Optimization Theory and Applications, Vol. 75, pp. 281-295, 1992.

2. Crouzeix, J. P., Pseudomonotone Variational Inequality Problems: Existence of Solutions, Mathematical Programming, Vol. 78, pp. 305314, 1997.

3. Hadjisavvas, N., and Schaible, S., Quasimonotone Variational Inequalities in Banach Spaces, Journal of Optimization Theory and Applications, Vol. 90, pp. 95-111, 1996.

4. Danillidis, A., and Hadjisavvas, N., On the Subdifferentials of Pseudoconvex and Quasiconvex Functions and Cyclic Monotonicity, Journal of Mathematical Analysis and Applications, Vol. 237, pp. 30-42, 1999.

5. Luc, D.T., Existence Results for Densely Pseudomonotone Variational Inequalities, Journal of Mathematical Analysis and Applications, Vol. 254, pp. 291-308, 2001.

6. Aussel, D., and Hadjisavvas, N., On Quasimonotone Variational Inequalities, Journal of Optimization Theory and Applications, Vol. 121, pp. 445-450, 2004.

7. Farouq, N.L., Convergent Algorithm Based on Progressive Regularization for Solving Pseudomonotone Variational Inequalities, Journal of Optimization Theory and Applications, Vol. 120, pp. 455-485, 2004.

8. Konnov, I. V., Partial Proximal Point Method for Nonmonotone Equilibrium Problems, Optimization Methods and Software, Vol. 21, pp. 373384, 2006.

9. Bai, M. R., Zhou, S. Z., and Ni, G. Y., Variational-like Inequalities with Relaxed $\eta-\alpha$ pseudomonotone Mappings in Banach Spaces, Applied Mathematics Letters, Vol. 19, pp. 547-554, 2006.

10. Bai, M.R., Zhou, S.Z., And Ni, G.Y., On Generalized Monotonicity of Variational Inequalities, Computers \& Mathematics with Applications, to appear.

11. FAn, K., A Generalization of Tychonoff's Fixed Point Theorem, Mathematische Annalen, Vol. 142, pp. 305-310, 1961. 
12. Aussel, D., Corvellec, J.N., and Lassonde, M., Mean Value Property and Subdifferential Criteria for Lower Semicontinuous Functions, Transactions of the American Mathematical Society, Vol. 347, pp. 4147-4161, 1995.

13. Aussel, D., Subdifferential Properties of Quasiconvex and Pseudoconvex Functions: Unified Approach, Journal of Optimization Theory and Applications, Vol. 97, pp. 29-45, 1998.

14. Penot, J.P., Are Generalized Derivatives Useful for Generalized Convex Functions? Generalized Convexity, Generalized Monotonicity, Edited by J.-P. Crouzeix, J.-E. Martínez-Legaz and M. Volle, Kluwer Academic Publishers, Dordrecht, pp. 3-59, 1998. 